\title{
GEOVISUALIZACIÓN DE LA POBLACIÓN: NUEVAS TENDENCIAS EN LA WEB SOCIAL ${ }^{1}$
}

\author{
Pablo Mateos ${ }^{2}$ \\ Centro de Investigaciones y Estudios Superiores en Antropología Social (CIESAS)

\section{RESUMEN}

La visualización geográfica, o geovisualización, sitúa al espacio como eje central en la exploración del conocimiento sobre el mundo que nos rodea. En este artículo se resumen algunas de las nuevas tendencias en la geoweb y la geovisualización de información sociodemográfica. Se presentan una serie de aplicaciones desarrolladas en proyectos de investigación en University College London (UCL), Reino Unido. Dichos proyectos de geovisualización tienen como fin hacer accesibles al público en general, representaciones alternativas de fenómenos sociales y poblacionales en el territorio. Para ello se facilita la contribución de contenido proveniente de diversas fuentes y sitios web, que puede ser integrado virtualmente a través de mapping mashups. Estos "agregados cartográficos" son configurados a gusto del usuario final sin ningún costo, permitiéndole compartir con el mundo su particular representación de la realidad socio-espacial en maneras inimaginables hace tan solo siete años.

Palabras clave: geovisualización, cartografía temática, censo de población, desigualdades urbanas, UCL-CASA.

\section{ABSTRACT}

\section{Geovisualization of populations: New trends in the social web}

Geographic visualization, or geovisualization, places space as the central theme in knowledge exploration about the world around us. This paper reviews recent trends in the geoweb and geovisualization of sociodemographic information. The paper presents several examples of research projects to geovisualize social and population dynamics developed at University College London (UCL). The main aim of these research projects on geovisualisation is to make available to the general public alternative representations of social and population phenomena over space. To this end, new geovisualisation technologies facilitate the combination of geographic content supplied from various sources and websites, but integrated together through mapping mashups. These "cartographic aggregations" are built to fit the end user's preferences at no cost. As a result, an average lay user can share with the world their particular sociospatial representations of reality in ways that seemed unthinkable just a few years ago.

Key words: geovisualization, thematic mapping, census of population, urban inequalities, UCL-CASA.

\section{INTRODUCCIÓN}

En los últimos diez años la geovisualización, o la representación visual de información geográfica, ha experimentado una auténtica revolución como resultado de una serie de factores técnicos y sociales que se abordan en este artículo.

\footnotetext{
Este artículo se ha elaborado dentro del proyecto de investigación "Contaminación atmosférica urbana y justicia ambiental: metodología de evaluación y estudio de casos con sistemas de información geográfica”, financiado por el Ministerio de Ciencia e Innovación de España, referencia CSO2011-26177.

2 pmateos@ciesas.edu.mx.
} 
Pese a los rápidos desarrollos de la cartografía digital entre las décadas de los 60 a los 90, e incluso su transposición en la web a partir de mitad de los 90, es solamente a partir de la mitad de la pasada década cuando se ha constatado una explosión de interés en el papel de la geografía como eje central en la exploración del conocimiento sobre el mundo que nos rodea. En efecto, "el mapa" se ha establecido rápidamente como una de las ventanas principales por las que el usuario medio de Internet se asoma a explorar el mundo que le rodea. Como factor crucial de este "renacimiento" de la geovisualización ha tenido mucho que ver la aportación de diversas formas creativas para la visualización del creciente volumen de información disponible por Internet, pero que parte de los ciudadanos y grupos pequeños en lugar de los Estados y las grandes corporaciones, los tradicionales productores de información geográfica. Este fenómeno ha sido denominado como la "democratización" de la información geográfica (Butler, 2006) y está caracterizado por formas muy innovadoras de geovisualización de información de muy distinta procedencia. En los últimos años, el ciudadano ordinario ha tomado un papel protagonista en la creación de representaciones alternativas de su entorno que desafien la legitimidad de las estadísticas y cartografías oficiales y denuncien las desigualdades socio-espaciales que le son palpables en su territorio inmediato.

En este artículo se argumenta que estos cambios en el tipo de agentes participantes, prácticas y técnicas de geovisualización de fenómenos sociales o naturales sobre el espacio, tienen una mayor trascendencia social que todos los desarrollos técnicos acaecidos en las décadas precedentes en el mundo de los Sistemas de Información Geográfica (SIG) y la cartografía. Dichas tendencias en geovisualización se han desarrollado de manera paralela al surgimiento de la web de segunda generación, también llamada web 2.0 o web social, que en el plano geográfico se ha venido a denominar la Geoweb (Elwood, 2009). A través de la Geoweb, los ciudadanos se han sentido rápidamente "empoderados" para explorar y expresar fenómenos geográficos de manera muy ágil e intuitiva. Dichos procesos han llevado a una rápida proliferación de habilidades básicas de interpretación espacial, que algunos autores han denominado la "alfabetización espacial" de la población. Sin embargo, los geógrafos y otros profesionales de la información geográfica, así como la academia y las instituciones gubernamentales en general, han llegado tarde a esta revolución en la democratización de la geovisualización. Éstos procesos realmente se han gestado en ámbitos mucho más cercanos a los usuarios finales y sus problemas de aplicación práctica, en gran parte resueltos por el amplio mundo de la informática, o las ciencias de la información sin geografía como adjetivo. Dicha transferencia del centro del protagonismo en la generación, adopción e interpretación de la información geográfica, desde "las élites" hacia los ciudadanos, tendrá una serie de importantes consecuencias futuras que apenas alcanzamos a vislumbrar en estos momentos. En este artículo intentaremos esbozar la dirección principal de dichos cambios a través de una serie de ejemplos ilustrativos de dicha trasformación tomados de primera mano en uno de los centros de investigación dónde se han gestado algunas de estas tendencias.

El objetivo de este artículo es doble. Por un lado, pretende sintetizar una serie de tendencias generales de índole técnica y social acaecidas en los últimos siete años en el mundo de la información geográfica y la geovisualización, como son la alfabetización espacial, la geoweb, y la información geográfica voluntaria, estableciendo las principales consecuencias de dichos cambios para el sector de la información geográfica. Por otro lado, se utilizan una serie de ejemplos de geovisualización innovadora de fenómenos socio-demográficos en el espacio para ilustrar dichas tendencias, establecer diferencias con los "usos y costumbres" anteriores, y acercar y promover el uso de dichas técnicas entre la comunidad de geógrafos y profesionales de la información geográfica de habla española.

En este artículo, en una primera parte (secciones 2, 3, y 4) se revisan dichas tendencias; desde la "información geográfica voluntaria" (volunteered geographic information) (Goodchild 2007) y la alfabetización de la población (spatial literacy), pasando por la geoweb y la geovisualización, para entrar de lleno en los denominados mapping mashups (que en este artículo traducimos como "agregados cartográficos") la principal técnica que ha facilitado esta transformación. En una segunda parte, el artículo presenta las consecuencias de estos desarrollos para la difusión de información socio-demográfica (sección 5), presentándose una serie de estudios de caso desarrollados por un equipo de geógrafos en University College London (UCL) (secciones 6, 7 y 8). Finalmente se presentan una serie de conclusiones que cierran el artículo. 


\section{LA "ALFABETIZACIÓN ESPACIAL" DE LA POBLACIÓN Y LA ERA DE LA "INFORMACIÓN GEOGRÁFICA VOLUNTARIA"}

El mundo en el que la información geográfica oficial fluía lentamente a través de costosas plataformas y complicados protocolos de intercambio de datos principalmente dirigidos a expertos de Sistemas de Información Geográfica (SIG), se derrumbó repentinamente en el verano de 2005, momento de aparición de los denominados "globos digitales" (Butler, 2006). Dichos globos en 3-D, tales como Google Earth o Microsoft Virtual Earth, y sus representaciones en mapas 2-D como Google Maps, Yahoo Maps, Bing Maps u OpenStreetMap, se han convertido rápidamente en un omnipresente "telón de fondo" geográfico sobre el cual los ciudadanos comunes y corrientes comparten de manera cotidiana todo tipo de contenido con algún componente espacial. Estos globos digitales y mapas en línea (online maps), forman parte de un conjunto de desarrollos tecnológicos acaecidos desde mediados de los años 2000, que han conformado un nuevo "ecosistema" denominado como la geoweb (Elwood, 2010a). La geoweb ha cambiado radicalmente el mundo de la información geográfica (IG) y su relación con la ciudadanía de a pie en cuestión de muy pocos años. A continuación se describen brevemente los avances socio-tecnológicos que han facilitando la revolución de la geoweb; la web 2.0, la información geográfica voluntaria, tecnologías de georeferenciación, mejoras en el procesamiento gráfico e internet de banda ancha.

La web 2.0 es un término genérico para definir colectivamente una serie de desarrollos en Internet que han cambiado profundamente a ésta a partir del año 2000. Se trata de un vuelco en la manera en la que se genera, disemina, circula y se consume información. En la primera generación de Internet, desde su aparición en torno a 1993 hasta el cambio de siglo, la información fluía "de arriba abajo", de manera estática y controlada por estructuras de poder que acreditaban la veracidad y calidad del contenido (gobiernos, empresas, instituciones civiles, ONGs, etc). El papel del usuario final era el de un consumidor pasivo de "contenido pre-verificado". Con la segunda generación de Internet, la Web 2.0, las distinciones entre productores y usuarios de información se han difuminado (Budhathoki et al., 2008). Cualquier usuario puede contribuir información en innumerables sitios compartiendo la tarea de proveer contenidos en Internet que otras personas encontrarán de utilidad. Esta filosofía "de abajo a arriba" está materializada por blogs, wikis, redes sociales, comentarios a páginas, videos tipo YouTube, periodismo ciudadano, y una infinidad de canales de comunicación diversos para proveer distintas versiones o representaciones de la realidad. La característica común de dicha revolución en la producción de información se ha venido a llamar el "contenido generado por usuarios" (user generated content), o por "la multitud" (crowd-sourcing), y un caso particular del mismo es el contenido geográfico. Goodchild (2007) ha acuñado el término "información geográfica voluntaria" (volunteered geographic information) o VGI, por sus siglas en inglés, para referirse a dicho contenido geográfico generado por los usuarios de manera altruista. La VGI se define como la extendida práctica de un gran numero de ciudadanos que se involucran a titulo particular, y generalmente con escasa formación, en la creación de información geográfica de manera voluntaria. De manera agregada, millones de colaboraciones cotidianas individuales hacen que la VGI esté llamada a alterar la generación de conocimiento geográfico, que hasta hace apenas una década era una tarea exclusiva de los gobiernos, concebido como recurso estratégico del Estado. Ejemplos de VGI son: plataformas abiertas (open source) de mapas como OpenStreetMap (OSM) o Wikimapia; fotos geocodificadas en Picasa, Flickr o Facebook; comentarios geocodificados; información de movilidad (p.ej. densidad de tráfico) capturada por cientos de personas como sensores a través del teléfonos móviles y GPS (Global Positioning System); e infinidad de datos sobre puntos de interés, medio ambiente, transporte publico, y un largo etc. que los ciudadanos suben a Internet con una localización sobre un mapa.

Otro pilar en el que se ha fundado la geoweb es la proliferación de tecnologías que facilitan enormemente la georeferenciación de información, es decir la descripción precisa de su localización espacial. Éstas son: el GPS, hoy en día omnipresente en teléfonos móviles, cámaras, ordenadores, coches, contenedores y muchos otros objetos; los callejeros digitales que permiten transformar una dirección postal o un topónimo en unas coordenadas geográficas, bien automáticamente o visualmente apuntando en un mapa; y la auto-ubicación de teléfonos móviles inteligentes (smartphones), que permiten geocodificar (geotagging) cualquier información que se sube a internet desde el mismo (por ejemplo, prácticas de geoblogging) 
Otros desarrollos que han facilitado la revolución de la geoweb son la mejora dramática en la capacidad de procesamiento gráfico de imágenes en los ordenadores, y el incremento en el acceso a Internet especialmente a través de banda ancha (ADSL en España). Estos dos aspectos por ejemplo fueron cruciales para el éxito de Google Earth, una tecnología que estaba ya disponible desde mitad de los años 90 pero solamente accesible por medio de super-ordenadores y redes de alta velocidad privadas (Goodchild, 2007).

Como resultado de estas tendencias, el conjunto de desarrollos conocidos como la Geoweb ha hecho que la mayoría de usuarios de internet se hayan acostumbrado rápidamente al manejo intuitivo de información geográfica, adquiriendo una cierta "alfabetización espacial" (spatial literacy) (Schultz et al., 2008). Mientras hasta hace apenas siete años, aquellos que utilizaban mapas en papel o por ordenador eran claramente una minoría, hoy aquel que no sabe leer mapas o interpretar información geográfica en la web sufre una "analfabetización espacial" que le impide acceder o entender gran parte de los contenidos que hoy circulan por Internet. En gran medida, la propagación de dicha alfabetización espacial ha sido facilitada por importantes desarrollos en el campo de la geovisualización.

\section{GEOVISUALIZACIÓN}

La visualización geográfica o geovisualización (GVis por sus siglas en inglés), se refiere a la representación visual de información geográfica para facilitar la comunicación de conocimiento espacial (Kraak, 2007). El campo más amplio de la visualización ha sido definido en español como "la transmisión de información y conocimiento a través de imágenes dirigidas a la vista" (Ojeda Zújar 2010, p.445), dentro del cual la geovisualización atañe a la información geográfica. Dichas definiciones desvelan su larga historia tanto en el mundo analógico (desde el origen de la cartografía) como en el digital (desde el origen de los SIG en los años 60). La principal ventaja de la geovisualización como herramienta de investigación es su utilidad en "todas las etapas de resolución de problemas en el análisis geográfico, desde el desarrollo de las hipótesis iniciales, hasta el descubrimiento de conocimiento, análisis, presentación y evaluación" (Buckley et al., 2000, p.2). No obstante, el valor de la geovisualización recae principalmente en la exploración de información geográfica y la comunicación de resultados, y no tanto en las etapas de análisis per se, el territorio tradicionalmente reservado al análisis espacial. Así, la geovisualización ofrece una manera sencilla de reducir la complejidad de grandes volúmenes de datos disponibles por Internet, o lo que se ha venido llamando el nuevo paradigma del Big Data. La geovisualización es clave para detectar patrones y relaciones en dichas masas de datos, permitiendo la búsqueda y recuperación de información incluso aunque inicialmente no se sepa muy bien lo que se está buscando (MacEachren y Kraak, 2001).

La geovisualización es un caso particular, una extensión espacial, del amplio campo de la visualización de información, es decir la representación visual y dinámica de la información para su mejor comprensión. Esta genealogía de la geovisualización ha sido generalmente ignorada por muchos profesionales de los SIG, que en gran medida han sido sobrepasados por las recientes tendencias de la geoweb y la geovisualización. Sin embargo, el espectacular desarrollo de la geovisualización en los últimos años no se entiende sin los desarrollos paralelos acaecidos en diversos campos como; informática, estadística, física, análisis visual (visual analythics), diseño (la infografía), procesamiento de gráficos (computer graphics) y los video-juegos. Es decir, la reciente revolución en geovisualización ha acaecido mayoritariamente de manera desconectada del nicho de los especialistas en SIG, tradicionalmente dominado por los profesionales de la Geografía, Ingeniería y otras ciencias sociales y medioambientales con un alto componente espacial.

Aunque los geógrafos han llegado tarde a este "renacimiento" de la geovisualización, se ha forjado un amplio consenso acerca del gran potencial que tiene la "geografía" (en minúsculas y en sentido amplio) para erigirse en el eje central de la visualización y exploración innovadora del conocimiento (Dodge, McDerby y Turner, 2006). Es decir, el mapa rápidamente se ha convertido en la ventana a través de la cual el ciudadano común y corriente interroga al mundo que le rodea para buscar y compartir información relevante. De ahí la justificación de la gran inversión en información geográfica que ha hecho el gigante Google, y el desarrollo de herramientas muy innovadoras para su exploración que pone gratuitamente a disposición del público. En otras palabras, la geovisualización, facilitada por la creciente alfabetización espacial de la ciudadanía, se torna en un interfaz clave para dar sentido al creciente volumen de información disponibles en Internet. 
La geovisualización no solamente consiste en representaciones espaciales de información cuantitativa a través de mapas (temáticos, físicos, de puntos, de superficies, fotografía aérea/satélite, etc), o incluso de cartogramas. Además de éstos, la geovisualización también incluye representaciones no cartográficas de datos cuantitativos con componentes espaciales (diagramas de redes, gráficos estadísticos, etc.), así como de material cualitativo que permite dotar de "significados" al espacio, como la fotografía, video, texto o audio con referencia explicita a su componente espacial. Elwood (2010b) ofrece un interesante resumen de las tendencias de la geovisualización de información cualitativa y multimedia en la geoweb. Finalmente, las recientes herramientas de geovisualización permiten sobre todo la combinación dinámica entre varias de estas representaciones visuales del espacio. Una herramienta clave para facilitar dichas combinaciones de geovisualizaciones es conocida como agregados cartográficos (mapping mashup). Ésta permite a un usuario común combinar geovisualizaciones de datos cualitativos o cuantitativos de muy diversa procedencia y calidad por las cuales navega sin ser consciente de debates entre epistemologías científicas y principios cartográficos. Es por esto que algunos han venido a llamar esta manera de entender la geovisualización y la geoweb como una nueva corriente ciudadana fuera de la ciencia; la neogeografía (Turner, 2006) o la "geografía de las masas" (Hudson-Smith et al., 2009), protagonizada por un público amateur de "cartógrafos profanos" (lay cartographers) (Elwood, 2010b). Aunque la elección del término neogeografía ha sido muy discutida (Leszczynski, 2010), el impacto de la revolución en la geovisualización acaecida en los últimos cinco años a través de los agregados cartográficos (mapping mashups), principalmente fuera de la academia, es innegable.

\section{MAPPING MASHUPS O AGREGADOS CARTOGRÁFICOS}

El principal desarrollo tecnológico que ha permitido la revolución en la geovisualización descrita en el anterior apartado, ha sido la posibilidad de combinar en la pantalla del usuario información geográfica de muy distinta procedencia sobre una base cartográfica estándar. Esta tecnología se conoce de manera general como agregados cartográficos (mapping mashups), literalmente un "conglomerado o mezcla de mapas". Este término ha sido adoptado rápidamente para referirse a las aplicaciones web de tipo hibrido que combinan información geográfica o aplicaciones de software de dos o más fuentes (Monmonier 2007). Estos websites tienen en común una nueva manera para facilitar no sólo el acceso a información geográfica sino la contribución de contenido georeferenciado proveniente del propio usuario o de diversas fuentes, todos ellos integrados virtualmente a través de mapping mashups. Dicho contenido puede incluir datos censales, estadísticas del gobierno, información de servicios comerciales, así como información geográfica voluntaria (VGI). Estos "collages cartográficos", son configurados a gusto del usuario final sin ningún costo, permitiéndole compartir con el mundo su particular representación de la realidad en maneras inimaginables hace tan solo unos años.

Su éxito se debe a una combinación de nuevas formas de visualización gráfica y acceso a la información geográficas de una manera dinámica que es rápida y fácilmente entendida por cualquier tipo de público. Lo que caracteriza esta tecnología es que está construida principalmente con una perspectiva de "abajo a arriba", en el que varios usuarios u organizaciones proporcionan contenido desde varios servidores y websites, en lugar de basarse en una estructura diseñada "de arriba a abajo" para difundir información geográfica de manera centralizada. En la Figura 1 se ofrece un diagrama de cómo se configura la información agregada en un mapping mashup. (Vid. Figura 1)

La tecnología de agregados cartográficos (mapping mashups) más utilizada tiene como base los mapas de Google Maps, un servicio web que "aumentó espectacularmente las expectativas de los usuarios con sus movimientos fluidos, una experiencia intuitiva y cartografía competente" (Fairhurst, 2005: 57). Además de los proveedores comerciales de globos digitales y mapas en línea ya citados, existe también una comunidad de software libre que ha tenido mucho éxito en el campo de la información geográfica, denominada OpenStreetMap (OSM) (www.openstreetmap.org) a la que se hará referencia más adelante. Aunque Google Maps no es una herramienta SIG como tal, la disponibilidad de una interfaz de programación de aplicaciones (API) estimula que los usuarios con conocimientos de programación básicos puedan construir sus propias aplicaciones usando Google Maps como un interfaz de visualización e integrando (o "mezclando" en la acepción del término mashup) sus propios datos geográficos. Por lo tanto, a los datos que 
proporciona el usuario, por ejemplo un mapa de puntos o mapa temático con alguna variable de interés, o fotografías, vídeos, audio y comentarios, se le da un marco geográfico fundamental, a través de cartografía de base muy detallada, que ayuda a situar y contextualizar la información temática presentada. Algunos se refieren a dicha cartografía base como "la geografía real", a diferencia de un SIG tradicional con un fondo "en blanco". Dicha cartografía de base incluye toponimia, límites administrativos, redes de carreteras y calles, puntos de interés, imágenes satélite y aéreas, y un largo etc., de manera estandarizada para todo el mundo, y con una granularidad (grado de detalle) que varía automáticamente según la escala (nivel de zoom). Antes de la aparición de Google Maps, los desarrolladores de mapas web tenían que dedicar mucho tiempo y un alto presupuesto a conseguir cartografía contextual relevante, o resolver complicados problemas de propiedad intelectual o proyecciones cartográficas respecto de la información geográfica adquirida en cada país o área de interés. Todo esto solo para tener un mapa base sobre el que mostrar los fenómenos que realmente interesaban. Además, las visualizaciones sobre Google Maps y Google Earth, y sus equivalentes en otros competidores, permiten al usuario cambiar la ubicación o la escala cartográfica de la visualización de una manera muy sencilla e intuitiva. Es decir, el usuario no tiene por qué saber de escalas cartográficas, proyecciones, shapefiles, códigos de hojas, simbología, así como niveles de cartografía administrativa (p.ej. distrito, código postal, asentamiento, municipio, provincia, etc) en cada país. El propósito central de los agregados cartográficos (mapping mashups) es por tanto que cualquier usuario con datos con alguna ubicación espacial (por ejemplo de GPS, de un teléfono móvil, o con direcciones, códigos postales o topónimos tomados de Internet) puede crear un mapa interesante en muy poco tiempo y sin ningún costo. Además, lo puede poner a disposición de otros usuarios que pueden incluso seguir añadir contenido al mismo a través de otras capas temáticas. Este hecho convierte a los API como Google Maps, o Google My Maps, en una herramienta para crear y publicar datos geográficos a través de un interfaz que se configura en una suerte de "tablón de anuncios global" (Barr, 2008).

Figura 1. Diagrama de un mapping mashup

\section{Mapping Mashups}

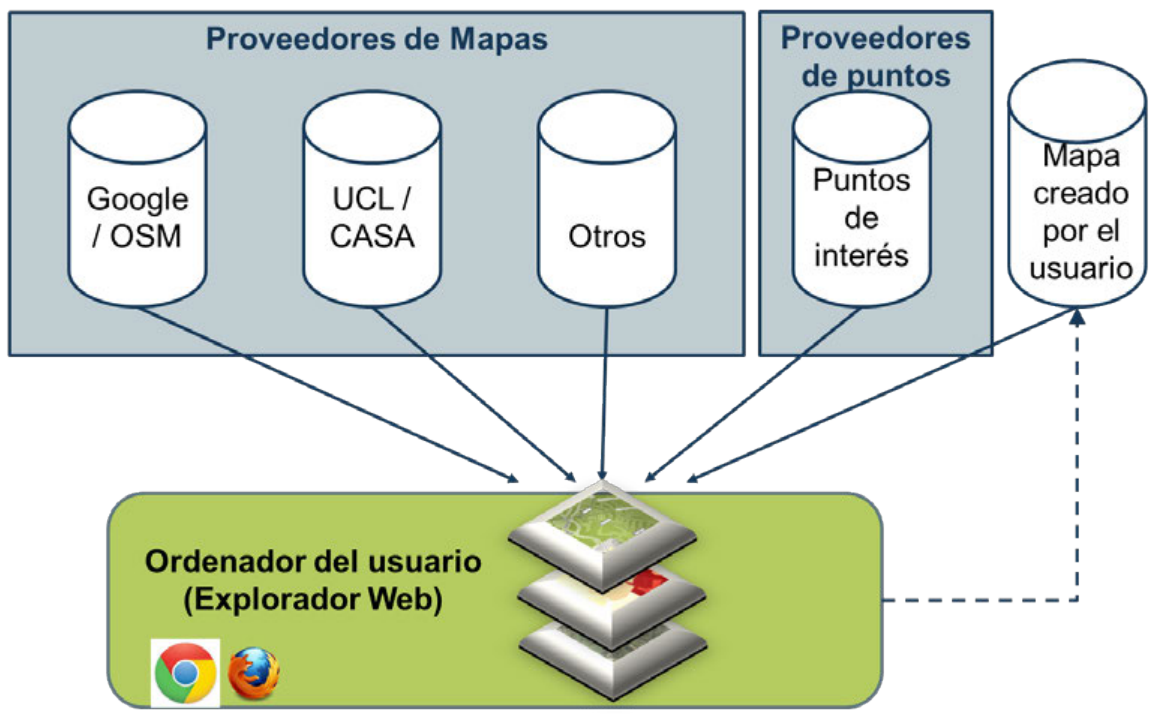

Clave: OSM= Open Street Map; UCL= University College London; CASA= Centre for Advanced Spatial Analysis

Finalmente, procede aclarar que la descripción de usuarios y la facilidad de creación de agregados cartográficos (mapping mashups) hecha en esta sección son necesariamente simplistas y homogeneizadoras. Obviamente existen varios tipos de "usuarios" de estas tecnologías. Distinguiremos aquí tres niveles generales; nivel 1 , aquellos usuarios con conocimientos básicos de programación, los cuales pueden crear sus propios agregados cartográficos (mapping mashups); nivel 2, un nivel intermedio de usuarios "hábiles", quienes pueden contribuir con nueva información (VGI), dependiendo de la facilidad dispuesta 
por el nivel 1 para ello; nivel 3, usuarios "básicos" que solamente "leen" el navegador, consumiendo la información disponible en los agregados cartográficos (mapping mashups). Esta estratificación de usuarios de la geoweb introduce nuevos mecanismos de exclusión, ya que solamente los usuarios del nivel 1 pueden crear sus propios agregados cartográficos (mapping mashups) (Elwood, 2009). No obstante, se puede argumentar que dichos factores son menos excluyentes que las barreras de acceso a la tecnología tradicional de los SIG cuyo coste de aprendizaje es mucho mayor y el nivel de especialización desalentaba a muchas personas de otras disciplinas con conocimientos de programación que ahora adoptan fácilmente las agregados cartográficos (mapping mashups). En cualquier caso los cambios de la última década descritos en el artículo, a través de la mencionada democratización de la información geográfica, han acelerado radicalmente las tendencias pre-existentes hacia el llamado "paradigma geotecnológico" (Buzai, 2001) o el "entendimiento geotecnológico de la cientificidad" (Moreno, 2013).

\section{GEOVISUALIZACIÓN DE INFORMACIÓN SOCIO-DEMOGRÁFICA}

En la mayoría de los países desarrollados, los medios de acceso a geovisualizaciones dinámicas de información socio-demográfica "oficial" no han estado a la altura de las cambiantes expectativas del ciudadano, que como ya vimos está "alfabetizado espacialmente". Las agencias estatales encargadas de producir y difundir las estadísticas oficiales sobre la población y el territorio, se han movido muy lentamente en el campo de la geovisualización. Pese a la universal presencia de la difusión por Internet, la dinámica para hacerlo suele ser muy estática y centralizada. Ésta consiste principalmente en dos metodologías; a) la búsqueda alfanumérica en una base de datos con la consecuente descarga de archivos pesados sin contexto; y b) mapas estáticos (imágenes) servidos por rígidas y lentas plataformas de SIG basadas en tecnologías caras y cerradas concebidas en los años 90 (por ejemplo ArcGIS server y estándares del Open GIS Consortium conocidos por las siglas WMS o WFS). Un ejemplo claro del vacío creado entre la geoweb y las geovisualizaciones oficiales de estadísticas de población, es el Censo de 2001 en el Reino Unido, cuyos mapas son accesibles a través del servicio Neighbourhood Statistics de la Oficina Nacional de Estadística (http://www.neighbourhood.statistics.gov.uk). Para acceder a un mapa de una variable censal es necesario contestar una serie de preguntas de cierto corte técnico y pasar por 11 pantallas hasta finalmente llegar a un mapa de una zona y resolución concreta. Si la combinación de elementos (geografía limítrofe, escala de desagregación, variable censal, método de clasificación, y esquema de color) no es la adecuada, hay que comenzar de nuevo y rehacer los 11 pasos. Esta filosofía de geovisualización diseñada en los años 90 y orientada a los especialistas del Censo, es totalmente inadecuada para diseminar los datos censales a las nuevas generaciones de usuarios de Internet, o la "Generación Google" (CIBER, 2008).

Dada esta clara necesidad, en los últimos cinco años se han desarrollado una serie de esfuerzos para llenar dicho vacío. Entre de ellos, destacan las aportaciones hechas por geógrafos de la población en el Reino Unido. En el resto de este este artículo se describen una serie de casos de estudio, centrados en aplicaciones de geovisualización sociodemográfica para explorar desigualdades espaciales en ámbitos urbanos. Se presentan diversos ejemplos de proyectos de investigación desarrollados en University College London (UCL) (Department of Geography y Centre for Advanced Spatial Analysis - CASA), como son MapTube, Gmap Creator, Census Profiler, y LondonProfiler entre otros. Todos estos proyectos de geovisualización tienen como fin hacer accesibles al público en general, representaciones alternativas y ágiles de fenómenos poblaciones en el territorio.

Con esta filosofía de abrir las herramientas de geovisualización a cualquier usuario, a partir de 2005 el Centre for Advanced Spatial Analysis (CASA) (http://www.bartlett.ucl.ac.uk/casa) de University College London (UCL) ha desarrollado una serie de sitios web con aplicaciones de agregados cartográficos (mapping mashups)que han tenido mucho éxito entre varios tipos de audiencias en varios países. Todas las herramientas mencionadas en adelante están adecuadamente documentadas a través de artículos de investigación (working papers) citados o documentación disponible en las páginas web que se aportan como referencia. En el artículo se omite cualquier tipo de digresión técnica, y aquellos lectores interesados pueden consultar más detalles en las referencias citadas para cada herramienta mencionada. 


\section{LOS COMIENZOS: GMAP CREATOR Y MAPTUBE}

Gmap Creator ${ }^{3}$ fue la primera herramienta desarrollada por CASA en 2005 para permitir a un usuario publicar un mapa temático sobre Google Maps. Gmap Creator es un programa que se puede descargar gratuitamente y requiere que los datos de entrada estén el formato cartográfico estándar denominado "shapefile". Dicho fichero ha de contener un mapa del área de interés con una división del espacio en zonas (representadas por polígonos), cada una de las cuales tiene asociados los datos de una o más variables correspondientes a dicha zona. Una vez importado dicho fichero shapefile en Gmap Creator, el usuario señala la variable a representar, el tipo de clasificación de datos en intervalos y el esquema de color para representar la variable. Como resultado Gmap Creator convierte el fichero vectorial tipo shapefile en una versión raster o imagen de dicho mapa, que posteriormente "trocea" en cientos o miles de teselas, de acuerdo con el formato de Google Maps. El número y tamaño de dichas teselas está determinado por la extensión del área de interés y los niveles de zoom (o de escala en la terminología cartográfica clásica) que se pretendan ofrecer al usuario final. Cada tesela se guarda como un archivo de imagen con formato JPEG con una nomenclatura específica que permite a Google Maps determinar su posición geográfica exacta y el nivel de zoom al que debe mostrarse. Finalmente el usuario de Gmap Creator decide dónde quiere guardar el nuevo mapa resultante y hacerlo disponible en la web. La posición de este mapa se indica en el diagrama de la Figura 1 como (UCL/CASA). El resultado final es un mapa temático disponible en la web, donde el mapa de fondo es el entorno estándar de Google Maps (mapa vectorial y /o mapa satélite) que reside en los servidores de Google, al cual se sobrepone el mapa temático que reside en los servidores del creador de este particular mashup. Ambos se mezclan únicamente al llegar al navegador del usuario final (ver Figura 1).

Para demostrar la utilidad de la superposición de diversas capas temáticas con esta arquitectura "ligera", se creó el sitio de Internet denominado "MapTube" (www.maptube.org). El nombre del mismo denota la idea de democratizar la publicación de información geográfica estableciendo un paralelismo con el popular sitio de publicación de videos YouTube. En este sitio se pueden subir mapas creados mediante Gmap Creator por diversos usuarios y superponerlos entre ellos formando una suerte de "meta-mashup". En la Figura 2 se ofrece un ejemplo de un mapa en MapTube donde se representan dos mapas aportados por usuarios, un mapa temático de marginalidad socioeconómica en Londres (primera capa) y un mapa de las líneas de metro (segunda capa), ambos sobre la cartografía base ofrecida por Google (tercera capa). Cabe enfatizar que estos tres mapas provienen de tres servidores y organizaciones distintas, y se juntan únicamente en el navegador del usuario final (ver diagrama de la Figura 1).

Figura 2. Ejemplo de visualización en MapTube; Mapa del índice de marginalidad y líneas de metro

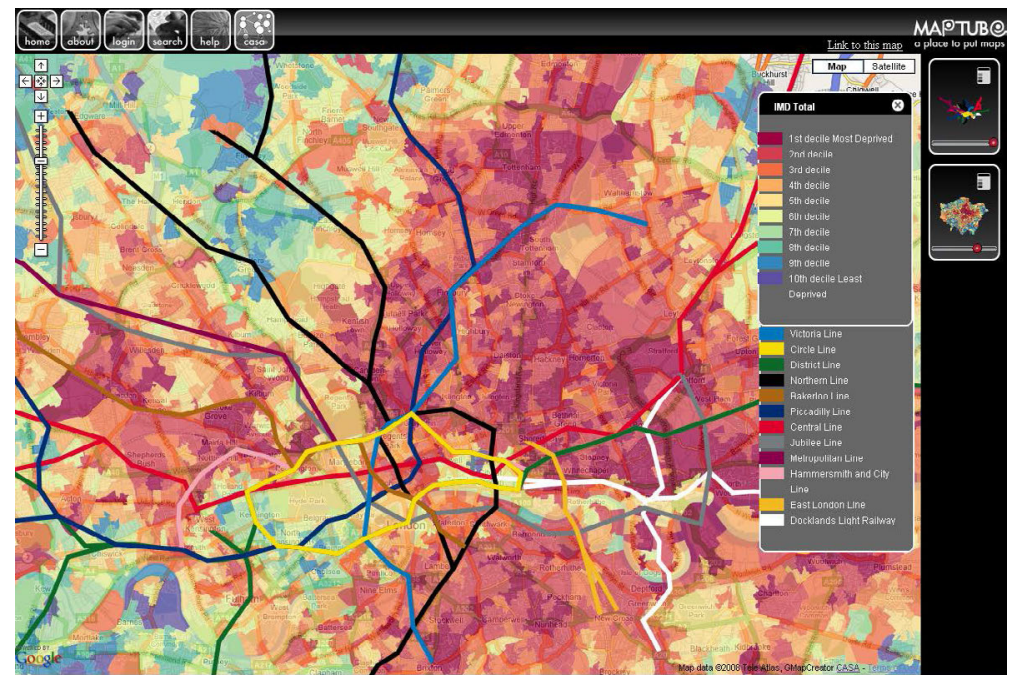

Fuente: www.maptube.org. Elaboración propia

3 Software disponible gratuitamente en http://www.bartlett.ucl.ac.uk/casa/latest/software/gmap creator 


\section{LONDOGNPROFILER - UN ATLAS SOCIOECONÓMICO DE LONDRES}

Utilizando la tecnología descrita en el anterior apartado, un equipo del departamento de Geografía de UCL (Gibin, Longley, Singleton y Mateos) desarrolló un atlas socioeconómico de Londres denominado London Profiler (www.londonprofiler.org). Los detalles acerca de cómo fue creado LondonProfiler pueden ser consultados en el artículo publicado por Gibin et al., (2008). En dicho atlas se representan diversos aspectos sobre la realidad socioeconómica de Londres utilizando datos de muy diversa procedencia. A continuación se describen los principales ámbitos temáticos y las referencias donde se puede encontrar más información sobre los mismos:

- Atlas Multicultural de Londres (Mateos et al., 2007)

- Clasificación de la sociedad de la información E-Society (Longley y Singleton, 2009)

- Clasificación del nivel educativo en el acceso a educación superior (Corver, 2005)

- Estadísticas de atención hospitalaria (www.hesonline.nhs.uk)

- Índice múltiple de marginalidad (Office of the Deputy Prime Minister, 2004)

- Clasificación de zonas en niveles socio-demográficos - Output Area Classification (Vickers and Rees, 2007)

- Mapa de accesibilidad en el transporte público (Transport for London:

http://data.london.gov.uk/datastore/package/public-transport-accessibility-levels)

- Mapa de niveles de crimen (http://www.police.uk/)

- Precios de vivienda (www.nestoria.com)

Cabe resaltar que todos estos ocho ámbitos contienen datos más recientes que aquellos publicados en el censo de población de 2001, el cual, a la hora de escribir estas líneas en Octubre de 2012, todavía es la última versión del censo disponible en el Reino Unido. Es decir, a través de la recopilación de distintas estadísticas y fuentes administrativas, de diversa índole y resolución espacial, se puede producir un atlas coherente de población sin la necesidad de esperar a los datos decenales del censo. Éste puede ser actualizado de manera continua a medida que dichos datos son actualizados por cada una de las fuentes.

Figura 3. LondonProfiler; mapa de puntos con viviendas en venta sobre un mapa temático con el índice de marginalidad en un barrio del oeste de Londres.

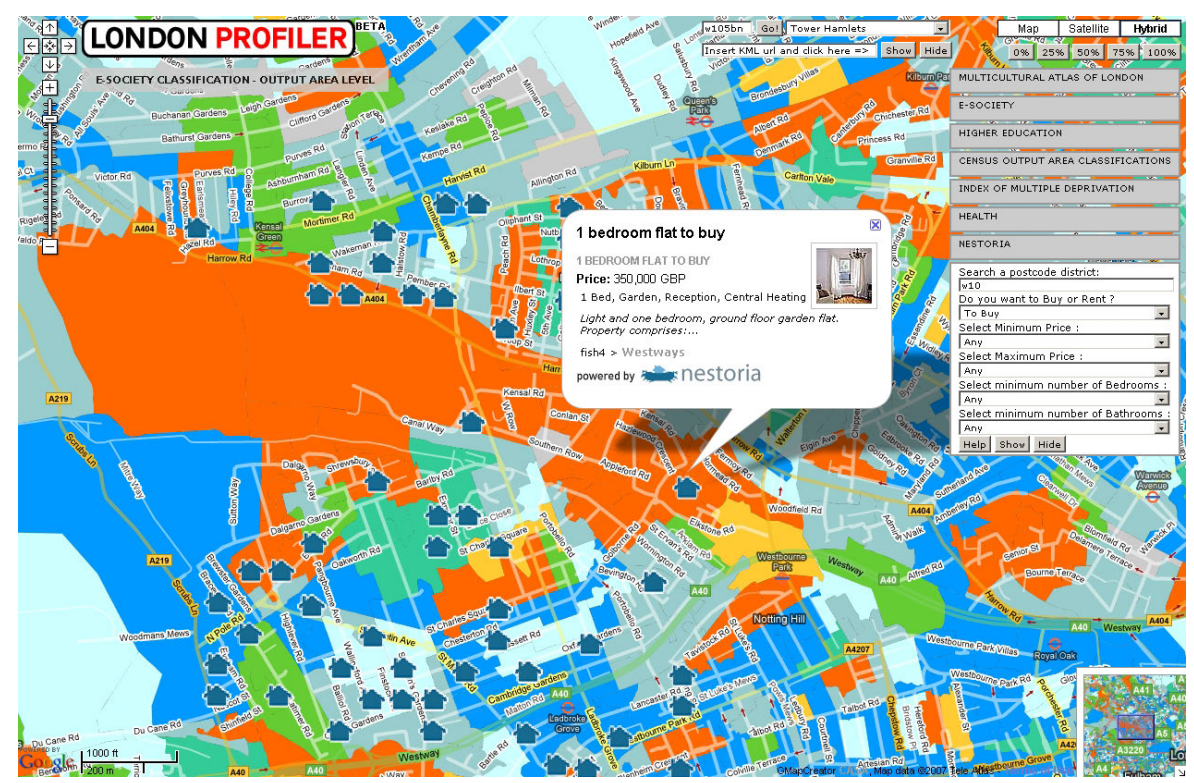

Fuente: www.londonprofiler.org. Elaboración propia en julio de 2008.

Otra de las novedades de LondonProfiler es la facilidad para conectarse con otros sitios web que proveen información geográfica a través del formatyo estándar KML de Google, y mostrarla sobre los mapas temáticos creados. Se ofrecen dos ejemplos en LondonProfiler. El primero es un servicio inmobiliario que ofrece información sobre viviendas en venta o alquiler por zonas de Londres llamado Nestoria (www.nestoria.com). A través de un simple menú el usuario de LondonProfiler selecciona 
un criterio de búsqueda de viviendas (rango de precio y tamaño) que es transmitido al website de Nestoria en tiempo real. En menos de dos segundos aparece una capa de puntos sobre el mapa temático sobreponiendo los inmuebles actualmente disponibles en el mercado que cumplen con dicho criterio de selección. Así, por ejemplo podemos explorar fotos y precios de viviendas junto con su localización y mapas socioeconómicos del entorno (índice de marginalidad, calidad escolar, servicios de salud, nivel de criminalidad, accesibilidad, etc.). Un ejemplo de este tipo de mapas se ofrece en la Figura 3. Finalmente, otro servicio en LondonProfiler permite al usuario "pegar" una dirección de internet (URL) donde previamente se haya colocado un archivo KML con algún mapa creado por el propio usuario. Es decir, se visualiza un mapa de puntos o de coropletas (temático) creado para algún uso específico, sobre los mapas generales socioeconómicos de LondonProfiler combinados con la cartografía de base de Google Maps. Esto permite que con unos pocos clics tengamos un mapa muy rico en detalle y contexto que puede ser creado por un usuario sin ninguna experiencia en SIG. Esto es precisamente lo que hemos hecho con estudiantes en la licenciatura de geografía tras un trabajo de campo donde recogieron información en una hoja de cálculo sobre productos de venta en tiendas de comestibles y que georeferenciaron y representaron en LondonProfiler junto con la distribución de ciertos grupos étnicos. Un ejemplo del resultado de dicho ejercicio se muestra en la Figura 4 (nótese parte de la dirección de internet en la franja superior central a la izquierda del botón de "Show". Esta es la ubicación del archivo creado por los alumnos en Google MyMaps).

Figura 4. Ejemplo del uso de LondonProfiler para un proyecto de campo con estudiantes de licenciatura.
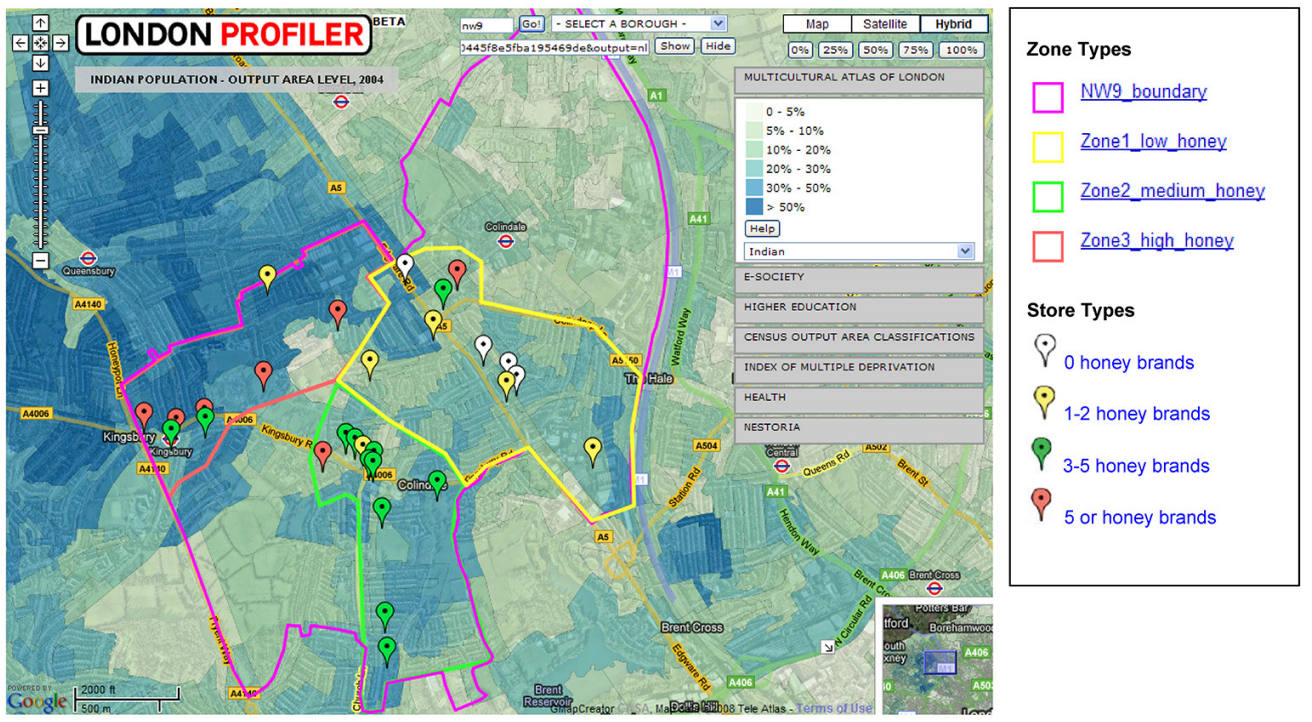

Fuente: Pablo Mateos y Paul Densham. Salida de campo con alumnos de primero de licenciatura en 2008.

\section{CENSUSPROFILER - GEOVISUALIZANDO EL CENSO DE POBLACIÓN PARA LA GENERACIÓN GOOGLE}

El ejemplo final presentado en este trabajo se refiere a los resultados de un proyecto de investigación titulado "Geovisualización de información geográfica del Censo", o Censusgiv por su acrónimo en inglés. Este proyecto desarrolló un nuevo sistema de geovisualización muy intuitivo con datos del Censo del Reino Unido, como prototipo para su posible uso en la divulgación de los datos del Censo de 2011. El prototipo se encuentra disponible en la página web www.censusprofiler.org y sirve aquí para demostrar el potencial de estas aplicaciones en la visualización geográfica del Censo y otros conjuntos de datos de población.

\footnotetext{
4 Proyecto de investigación financiado por el Economic and Social Research Council (ESRC) en el marco del Programa de Desarrollo del Censo (número de beca RES-348-25-0015)
} 
A finales de la década de los 2000, la oficina nacional de estadísticas del Reino Unido y la comunidad científica usuaria de datos de población eran conscientes de que en el campo del censo y otros conjuntos de datos administrativos de la población se estaba "perdiendo el tren" de las innovaciones en geovisualización en la era de la Web 2.0. El censo de 2001, hace once años, llevó a la elaboración de un conjunto de herramientas en línea que proporcionaban un acceso a una gama cada vez mayor de productos de datos digitales de libre disposición de la Oficina Nacional de Estadísticas (ONS). Sin embargo, los medios para visualizar y analizar la dimensión espacial de estos conjuntos de datos todavía hoy en 2012 sólo están disponibles para unos pocos investigadores que tienen un conocimiento profundo de SIG. Esto es necesario para poder vincular datos alfanúmericos con la cartografía digital que se distribuye por separado. A raíz de esta situación, en 2008 un equipo de geógrafos de University College London (UCL) consiguió financiación para un proyecto que podría ayudar a remediar esta situación, proyecto que dio como resultado la plataforma CensusProfiler.

Dicho proyecto partió de la premisa de que, para lograr una mayor difusión de los resultados del Censo de 2011, las visualizaciones geográficas del mismo se deberían construir en una plataforma que girara en torno a un mapa como elemento de partida (en lugar de a una serie de consultas en una base de datos). Dicho mapa de inicio, debería mostrar la geografía real de una zona, y no un mapa en blanco o administrativo de zonas censales. Así, los usuarios se familiarizarían rápidamente con la información de la zona y la interfaz de usuario. Es decir, la herramienta de difusión de datos no supondría un obstáculo entre el usuario y la información geográfica. A partir de dicho mapa el usuario puede sumergirse en niveles de zoom con mucho más detalle, moverse rápidamente a otras zonas de la ciudad o del país, o cambiar de variable censal una vez que haya encontrado una zona de interés, e incluso añadir otra información. Todo esto utilizando el estilo de navegación intuitiva del Google Maps y Google Earth.

El resultado final con el prototipo de geovisualización del Censo de 2011 (con datos de 2001) puede ser explorado en www.censusprofiler.org. Este fue realizado con una tecnología distinta a la descrita anteriormente. Es decir, se prescindió de Google como proveedor de información geográfica base, sustituyéndose por OpenStreetMap (OSM), una plataforma de carácter abierto y gratuito. Dicha cartografía se puede descargar y transformar libremente eligiendo qué elementos y cómo han de ser representados en el mapa base. Asimismo, se confeccionó una arquitectura basada en el software abierto y libre lo cual facilita su popularización y adaptación por futuros desarrolladores en éste área. La descripción técnica completa detrás de CensusProfiler se encuentra disponible en Mateos y O'Brien (2011). Un ejemplo del aspecto del sitio web se muestra en la Figura 5.

Figura 5. Pantalla principal del CensusProfiler, prototipo de geovisualización del Censo del Reino Unido.

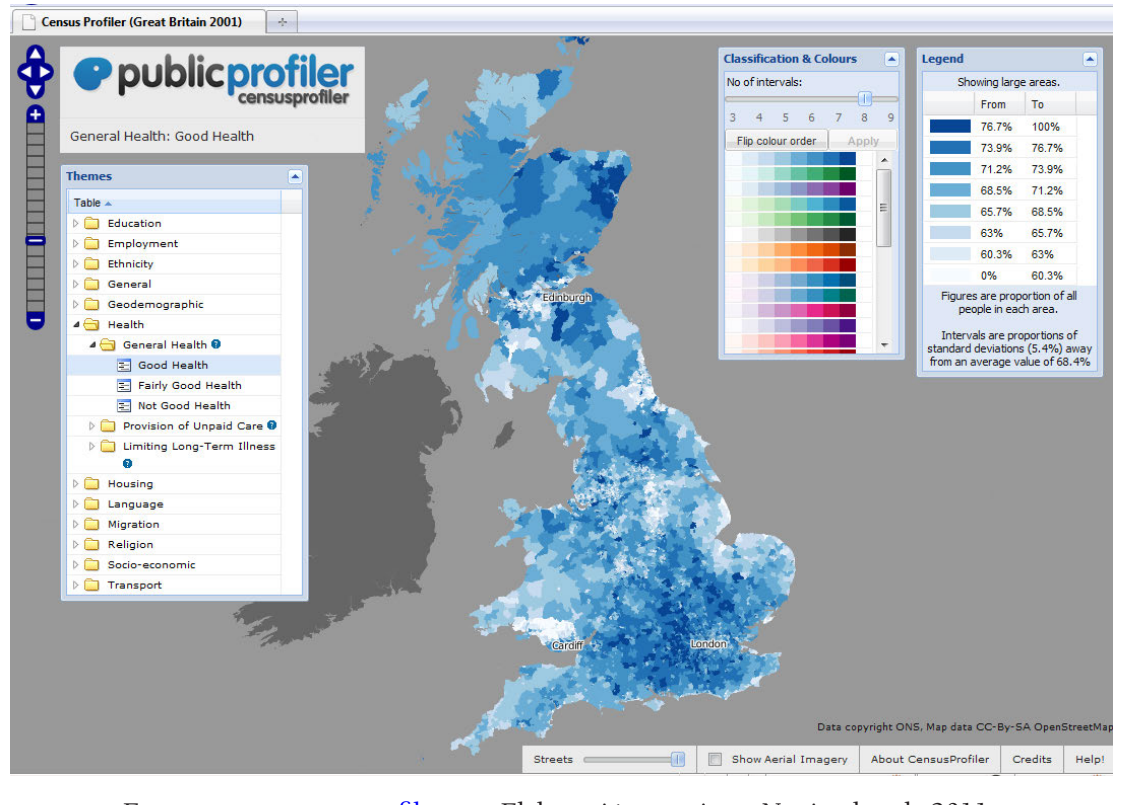

Fuente: www.censusprofiler.org. Elaboración propia en Noviembre de 2011. 
La ventaja principal de CensusProfiler es la facilidad de navegación (panning y zooming) y lo intuitivo de las decisiones a tomar para llegar a un mapa interesante. En otras palabras, el mapa es la ventana para interrogar los datos del censo y no el resultado final. Éste tipo de geovisualización no interfiere en la exploración de los datos, ya que decisiones como el nivel de desagregación geográfica de los datos censales (tamaño de zonas o distritos), el grado de detalle en la cartografía base de contexto, o el método de clasificación en intervalos para el mapa temático son tomadas automáticamente por el sistema. El primer y segundo factor dependen de la escala de observación (nivel de zoom), mientras que el tercer factor del tipo de variable seleccionada. A partir de ese punto, una vez que el usuario está satisfecho con la zona elegida, puede cambiar muy fácilmente cuatro elementos; la variable censal, el número de intervalos en los que se clasifica dicha variable, el esquema de color con el que se representan y la cartografía base.

Con este mapping mashup, cualquier persona puede crear sus propias geovisualizaciones del censo de población de manera dinámica y sin seguir recetas previas. Los contenidos de CensusProfiler pueden ser "envueltos" dentro de otros mashups, agregando todo tipo de información que el usuario considere relevante para su particular representación, temática o zona de interés. Finalmente, la tecnología de software abierto permite que este sitio web pueda ser reproducido, mejorado o utilizado como material base para desarrollar otras plataformas, sin estar restringidos por el API de Google como sus decisiones sobre el diseño cartográfico o estrategia publicitaria sobre sus mapas.

\section{CONCLUSIÓN}

Durante la última década, una serie de nuevas tendencias en la creación de la geoweb y el desarrollo de métodos innovadores de geovisualización han alterado profundamente las expectativas del público en general acerca de la divulgación de información sociodemográfica con un componente espacial. Estas tendencias están protagonizadas por: a) la disponibilidad masiva de datos con contenido geográfico, sobre los que el Estado y las instituciones formales ya no tienen competencia exclusiva, y b) el desarrollo de nuevas herramientas de geovisualización, que facilitan su combinación, consulta, manipulación y participación ciudadana. En este artículo hemos revisado cómo la geoweb y las nuevas técnicas de geovisualización, a través de la información geográfica voluntaria y los agregados cartográficos (mapping mashups) han facilitado la "alfabetización espacial" de la ciudadanía.

A través de servicios como Google Maps y OpenStreetMap se han creado plataformas con una detallada cartografía de todo el mundo, conformándose en un telón de fondo global sobre el que el ciudadano comparte y consume geo-información. Es más, dichas plataformas permiten a un usuario con un nivel de conocimiento de informática muy básico, la configuración de servicios de geovisualización de información sobre el territorio que eran impensables hace tan solo siete años. Los mapping mashup, permiten a usuarios con distintos niveles de habilidad, combinar capas de información y mapas provenientes de organizaciones muy diversas y con muy distintos protocolos técnicos, escalas y resoluciones espaciales de la información. El resultado son "collages" de mapas temáticos superpuestos, que aportan un importante valor agregado para el análisis exploratorio de fenómenos espaciales. Todo esto se puede realizar con un coste muy bajo y en poco tiempo. Este estado de la cuestión, presenta una gran diferencia con la era de los grandes y costosos servidores de información SIG de hasta hace apenas unos años, donde se ofrecían servicios mucho más restringidos de geovisualización a través de caros y lentos proyectos de distribución de información centralizada.

Se han presentado diversos ejemplos de este tipo de geovisualizaciones de datos sociodemográficos en el Reino Unido, desarrollados en University College London, (UCL) (Department of Geography y Centre for Advanced Spatial Analysis - CASA). Aquí hemos mencionado; Gmap creator, MapTube, LondonProfiler y Census Profiler, pero existen muchos otros ejemplos en el mundo de este tipo de tecnología creciente que supone la democratización del acceso a la información geográfica. En muchos casos, el propio usuario puede crear su propia versión de la realidad a través de nuevas interpretaciones alternativas sobre las estadísticas poblacionales para denunciar desigualdades socio-espaciales, dotándole de herramientas por ejemplo para enfrentarse a una intervención de obra pública en su zona, o cuestionar decisiones de recortes de servicios públicos en determinado barrio de acuerdo a su perfil sociodemográfico dentro de la ciudad. Algunos otros ejemplos aparecen en la Figura 6 y muchos otros están disponibles en la página web de 
CASA (http://www.bartlett.ucl.ac.uk/casa). A través de este artículo, esperamos haber sembrado una serie de ideas que permitan a los geógrafos y demógrafos de habla española adoptar y adaptar estas tecnologías de geovisualización para democratizar y popularizar el acceso a la información sociodemográfica fuera del mundo académico y los profesionales SIG. El futuro claramente estará dominado por ciudadanos con una "alfabetización espacial" mucho mayor que la de generaciones anteriores y para los que el mapa es una ventana mediadora entre el mundo real y el virtual entre los que se mueven cómodamente. Es nuestra tarea "devolverles" la información que recolectamos sobre ellos de manera mucho más equitativa y participativa, facilitando su "empoderamiento" en el saber sobre el territorio, mediante el concurso de estas tecnologías geográficas.

Figura 6. Ejemplos de vistas alternativas de clasificaciones de población sobre OpenStreetMap (izquierda) y Google Earth (derecha), utilizando la tecnología desarrollada en CensusProfiler.

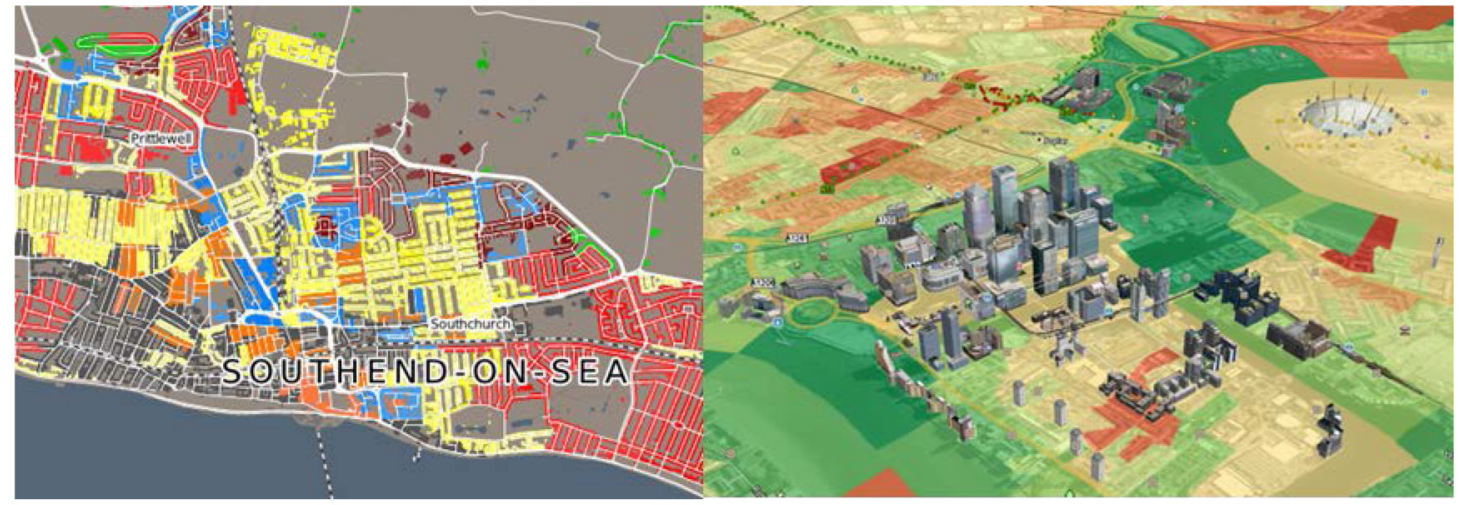

Fuente: Oliver O’Brien. Reproducidos con permiso del autor.

\section{BIBLIOGRAFÍA}

BARR, R. (2008): Google and GI science: Revolution, evolution or wild goose? London: Centre for Advanced Spatial Analysis Seminar, University College London, 30th January 2008.

BUCKLEY, A. (2004): Geographic Visualization. In Mcmaster, R. B. and Usery, E. L. A Research Agenda for Geographic Information Science. Boca Raton, Fl: Crc Press: pp. 270 - 296.

BUCKLEY, E., GAHEGAN, M. \& CLARKE, K. C. (2000): Geographic visualization as an emerging research theme in GIScience. Santa Barbara, CA.

BUDHATHOKI, N., BRUCE, B. y NEDOVIC-BUDIC, N. (2008): Reconceptualizing the role of the user of spatial data infrastructures. GeoJournal, 72, pp. 149-60.

BUTLER, D. (2006): Virtual globes: The web-wide world. Nature, 439, pp. 776 - 778.

BUZAI, G. D. (2001): "Paradigma Geotecnológico, Geografía Global y CiberGeografía, la gran explosión de un universo digital en expansión", GeoFocus (Artículos), 1, pp. 24 - 48.

CIBER (2008): Information behaviour of the researcher of the future. A report commissioned by The British Library and JISC. University College London. Disponible en: http://www.jisc.ac.uk/media/documents/ programmes/reppres/gg_final_keynote_11012008.pdf

CORVER, M. (2005): Young participation in Higher Education. HEFCE 2005/03. Bristol: HEFCE.

DODGE, M., MCDERBY, M. \& TURNER, M. (2006): The power of geographical visualizations. In Geographic Visualization: Concepts, Tools and Applications. New York: Wiley.

ELWOOD, S:

- (2009): Geographic information science: new geovisualization technologies-emerging questions and linkages with Giscience research. Progress in Human Geography, 33(2), pp. 256 - 263.

- (2010a): Geographic information science: visualization, visual methods, and the geoweb. Progress in Human Geography, 35(3), pp. 401 - 408. 
- (2010b): Geographic information science: emerging research on the societal implications of the geospatial web. Progress in Human Geography, 34(3), pp. 349-357.

FAIRHURST, R. (2005): Next-generation web mapping. SoC (Society of Cartographers) Bulletin, 39, pp. $57-61$.

GIBIN, M., SINGLETON, A., MILTON, R., MATEOS, P. y LONGLEY, P. (2008): Collaborative Mapping of London Using Google Maps: The LondonProfiler. CASA Working Papers Series 132. Disponible en: http://www.bartlett.ucl.ac.uk/casa/pdf/paper132.pdf

GOODCHILD, M. F. (2007): Citizens as sensors: the world of volunteered geography. GeoJournal, 69(4), pp. $211-221$

HUDSON-SMITH, A., BATTY, M., CROOKS, A. \& MILTON, R. (2009): Mapping for the masses: accessing web 2.0 through crowdsourcing. Social Science Computer Review, 27(4), pp. 524-538.

KRAAK, M. (2007): Geovisualization and visual analytics. Cartographica: The International Journal for Geographic Information and Geovisualization, 42(2), pp. 115-116.

LESZCZYNSKI, A. (2010): Neogeo as neolib? Towards a preliminary political economy of VGI. Paper presented at the Annual Meeting of the Association of American Geographers, Washington, DC, 15 April.

LONGLEY, P.A. \& SINGLETON, A.D. (2009): Classification through consultation: public views of the geography of the e-society. International Journal of Geographical Information Science, 23(6), pp. 737-763.

MACEACHREN, A. y KRAAK, M. (2001): Research challenges in geovisualization. Cartography and Geographic Information Systems 28, pp. 3-12.

MATEOS, P. and O'BRIEN, O. (2011): CensusProfiler - Creating accessible Geovisualizations of the Census of Population, CASA Working Paper 174. University College London. Disponible en http://www. bartlett.ucl.ac.uk/casa/pdf/paper174.pdf

MATEOS, P., WEBBER, R. \& LONGLEY, P. A. (2007): The Cultural, Ethnic and Linguistic Classification of Populations and Neighbourhoods using Personal Names. CASA Working Paper 116. Disponible en: http://www.bartlett.ucl.ac.uk/casa/publications/working-paper-116

MONMONIER, M. (2007): Cartography: the multidisciplinary pluralism of cartographic art, geospatial technology, and empirical scholarship. Progress in Human Geography, 31(3), 371 p.

MORENO, A. (2013): Entendimiento y naturaleza de la cientificidad geotecnológica: una perspectiva desde el pragmatismo epistemológico, Investigaciones Geográficas, [este número]

OFFICE OF THE DEPUTY PRIME MINISTER (2004): Index of Multiple Deprivation (online). Disponible en: http://data.gov.uk/dataset/imd 2004. Accessed 18th December 2007. London: Department of Communities and Local Government.

OJEDA, J. (2010): Geovisualización: espacio, tiempo y territorio. Ciudad y territorio: Estudios territoriales.165-166; pp. 445 - 460.

SCHULTZ, R. B., KERSKI, J.J. \& PATTERSON, T. C. (2008): The use of virtual globes as a spatial teaching tool with suggestions for metadata standards. Journal of Geography, 107(1), pp. 27 - 34.

TURNER, A. (2006): Introduction to Neogeography, O'Reilly. http://www.oreilly.com/catalog/neogeography/

VICKERS, D. y REES, P. (2007): Creating the National Statistics 2001 Output Area Classification, Journal of the Royal Statistical Society, Series A 170(2). 